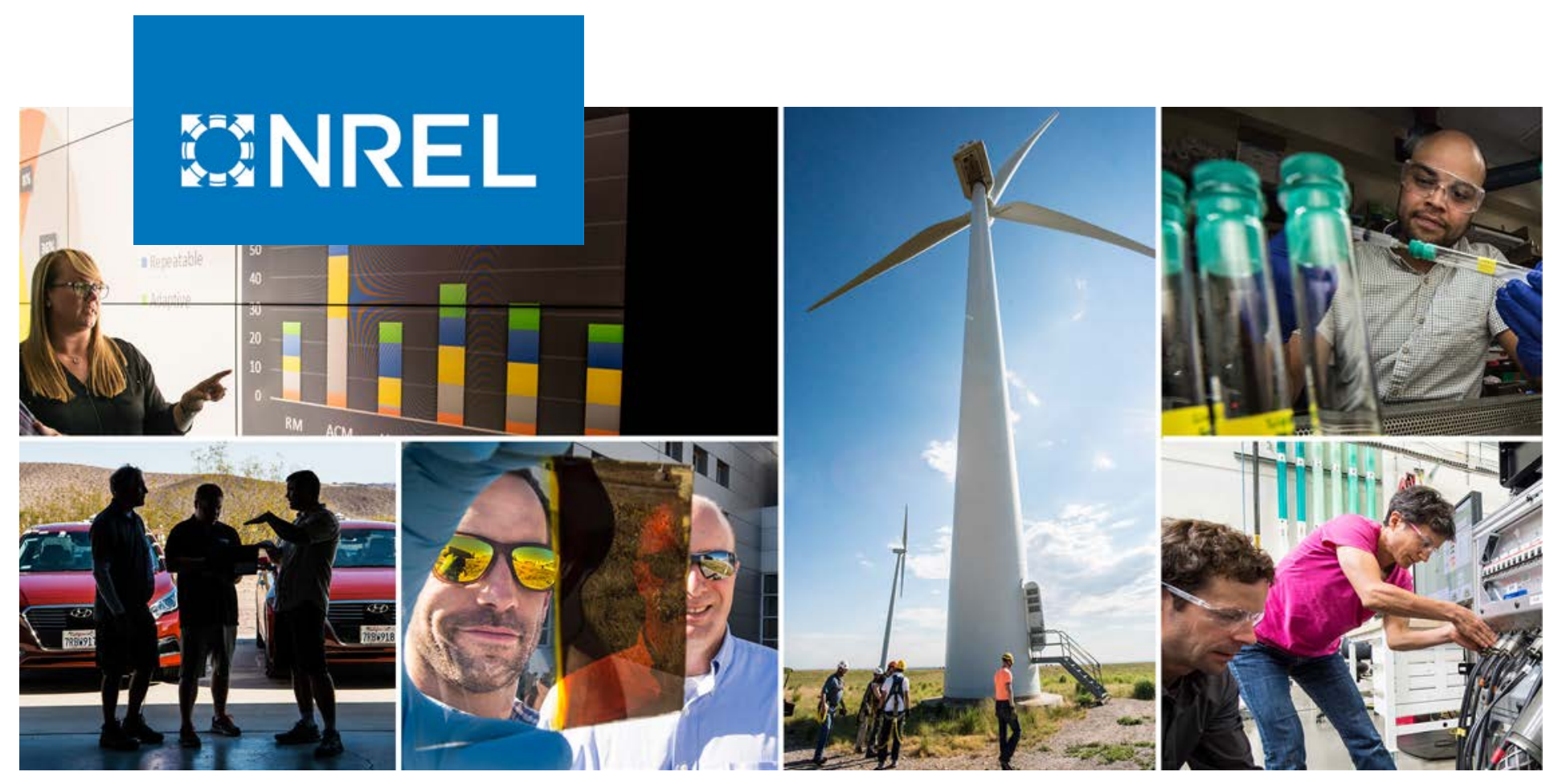

\title{
Enabling Realistic Communications Evaluations for ADMS
}

Emma Raszmann, ${ }^{1}$ Kumaraguru Prabakar, ${ }^{1}$ Soumya Tiwari, ${ }^{1}$ Ismael Mendoza, ${ }^{1}$ Harsha Padullaparti, ${ }^{1}$ Barry Mather, ${ }^{1}$ Deepthi Vaidhynathan, ${ }^{1} \mathrm{Jim} \mathrm{Li},{ }^{2}$ Mike Brozek, ${ }^{2}$ and Mar Tarres ${ }^{2}$

1 National Renewable Energy Laboratory

2 Anterix

NREL is a national laboratory of the U.S. Department of Energy Office of Energy Efficiency \& Renewable Energy

Operated by the Alliance for Sustainable Energy, LLC

This report is available at no cost from the National Renewable Energy Laboratory (NREL) at www.nrel.gov/publications.
Technical Report

NREL/TP-5D00-80474

September 2021 


\section{GNREL}

\section{Enabling Realistic Communications Evaluations for ADMS}

Emma Raszmann, ${ }^{1}$ Kumaraguru Prabakar, ${ }^{1}$ Soumya Tiwari, ${ }^{1}$ Ismael Mendoza, ${ }^{1}$ Harsha Padullaparti, ${ }^{1}$ Barry Mather, ${ }^{1}$ Deepthi Vaidhynathan, ${ }^{1} \mathrm{Jim} \mathrm{Li},{ }^{2}$ Mike Brozek, ${ }^{2}$ and Mar Tarres ${ }^{2}$

1 National Renewable Energy Laboratory 2 Anterix

\section{Suggested Citation}

Raszmann, Emma, Kumaraguru Prabakar, Soumya Tiwari, Ismael Mendoza, Harsha Padullaparti, Barry Mather, Deepthi Vaidhynathan, Jim Li, Mike Brozek, and Mar Tarres. 2021. Enabling Realistic Communications Evaluations for ADMS. Golden, CO: National Renewable Energy Laboratory. NREL/TP-5D00-80474.

https://www.nrel.gov/docs/fy21osti/80474.pdf.

NREL is a national laboratory of the U.S. Department of Energy Office of Energy Efficiency \& Renewable Energy Operated by the Alliance for Sustainable Energy, LLC

This report is available at no cost from the National Renewable Energy Laboratory (NREL) at www.nrel.gov/publications.

Contract No. DE-AC36-08GO28308
Technical Report

NREL/TP-5D00-80474

September 2021

National Renewable Energy Laboratory 15013 Denver West Parkway Golden, CO 80401 303-275-3000 • www.nrel.gov 


\section{NOTICE}

This work was authored in part by the National Renewable Energy Laboratory, operated by Alliance for Sustainable Energy, LLC, for the U.S. Department of Energy (DOE) under Contract No. DE-AC36-08GO28308. Funding provided by the U. S. Department of Energy Office of Energy Efficiency and Renewable Energy via the Energy Systems Integration Facility. The views expressed herein do not necessarily represent the views of the DOE or the U.S. Government.

This report is available at no cost from the National Renewable Energy Laboratory (NREL) at www.nrel.gov/publications.

U.S. Department of Energy (DOE) reports produced after 1991 and a growing number of pre-1991 documents are available free via www.OSTI.gov.

Cover Photos by Dennis Schroeder: (clockwise, left to right) NREL 51934, NREL 45897, NREL 42160, NREL 45891, NREL 48097, NREL 46526.

NREL prints on paper that contains recycled content. 


\section{List of Acronyms}

4G

$5 \mathrm{G}$

ADMS

AMI

DER

DERMS

DOE

DTT

DVR

GOOSE

HAN

IEC

IEEE

LTC

LTE

NAN

NREL

PLM

PV

RSSI

RTAC

SCADA

SEL

TCP

WAN

WiMAX fourth $\left(4^{\text {th }}\right)$ generation

fifth $\left(5^{\text {th }}\right)$ generation

advanced distribution management system

advanced metering infrastructure

distributed energy resource

distributed energy resource management system

U.S. Department of Energy

direct transfer trip

dynamic voltage regulation

Generic Object-Oriented Substation Event

home-area network

International Electrotechnical Commission

Institute of Electrical and Electronics Engineers

load tap changer

Long-Term Evolution

neighbor-area network

National Renewable Energy Laboratory

peak load management

photovoltaic

received signal strength indication

real-time automation controller

supervisory control and data acquisition

Schweitzer Engineering Laboratories

Transmission Control Protocol

wide-area network

Worldwide interoperability for microwave access 


\section{Executive Summary}

Increasing penetration levels of inverter-based distributed energy resources (DERs) in distribution systems are creating challenges in system operation. Currently, wired or proprietary wireless communications are used by advanced distribution management systems (ADMS) to detect the state of the system and to control the DERs for optimal operation. Wireless communications are an alternative solution that can enable connectivity between the dispersed assets used in distribution system applications. As demand for the seamless integration of DERs continues to increase, proper networking and communication systems are becoming important to support the DER infrastructure.

This report summarizes the validation of a private wireless Long-Term Evolution (LTE) network in a laboratory environment. The purpose of this work is to (1) demonstrate the effectiveness of private wireless LTE communications for grid applications and to (2) provide a means of evaluating latency and overall performance of wireless communications in grid applications.

In the first phase of this work, private wireless LTE communications were validated in a direct transfer trip (DTT) relaying scenario as an example of one-way communications using grid equipment. This study provides a baseline understanding of the variation in latency for seven different kinds of wireless scenarios, shown in Figure ES-1. As expected, weak (attenuated) wireless signal-based communications yielded the widest ranges in latency. When higher priority was set for the trip signal communications, this resulted in lower latency in communication timing.

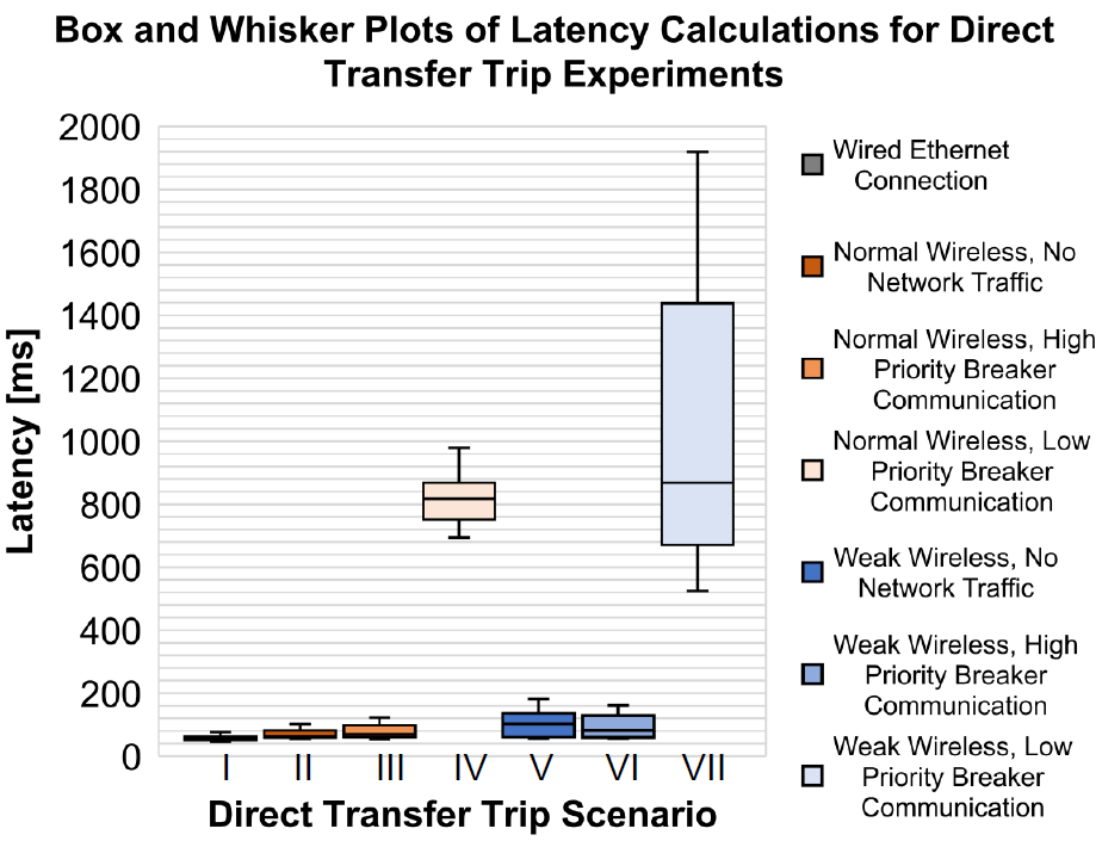

Figure ES-1. Box and whisker plots to show trends in latency calculations across all 70 DTT experiments (inclusive median)

In the final phase of this project, the private wireless LTE network was validated using two-way communications in an ADMS test bed that was developed at the Energy Systems Integration Facility at the National Renewable Energy Laboratory. The objective was to study the impact of 
wireless communications on grid performance, particularly in this study for peak load management applications. This study expands on the findings shown in the first phase of this project by demonstrating the range in timing for wired, wireless, and weak (attenuated) wireless communication signals in a controller-hardware-in-the-loop test bed.

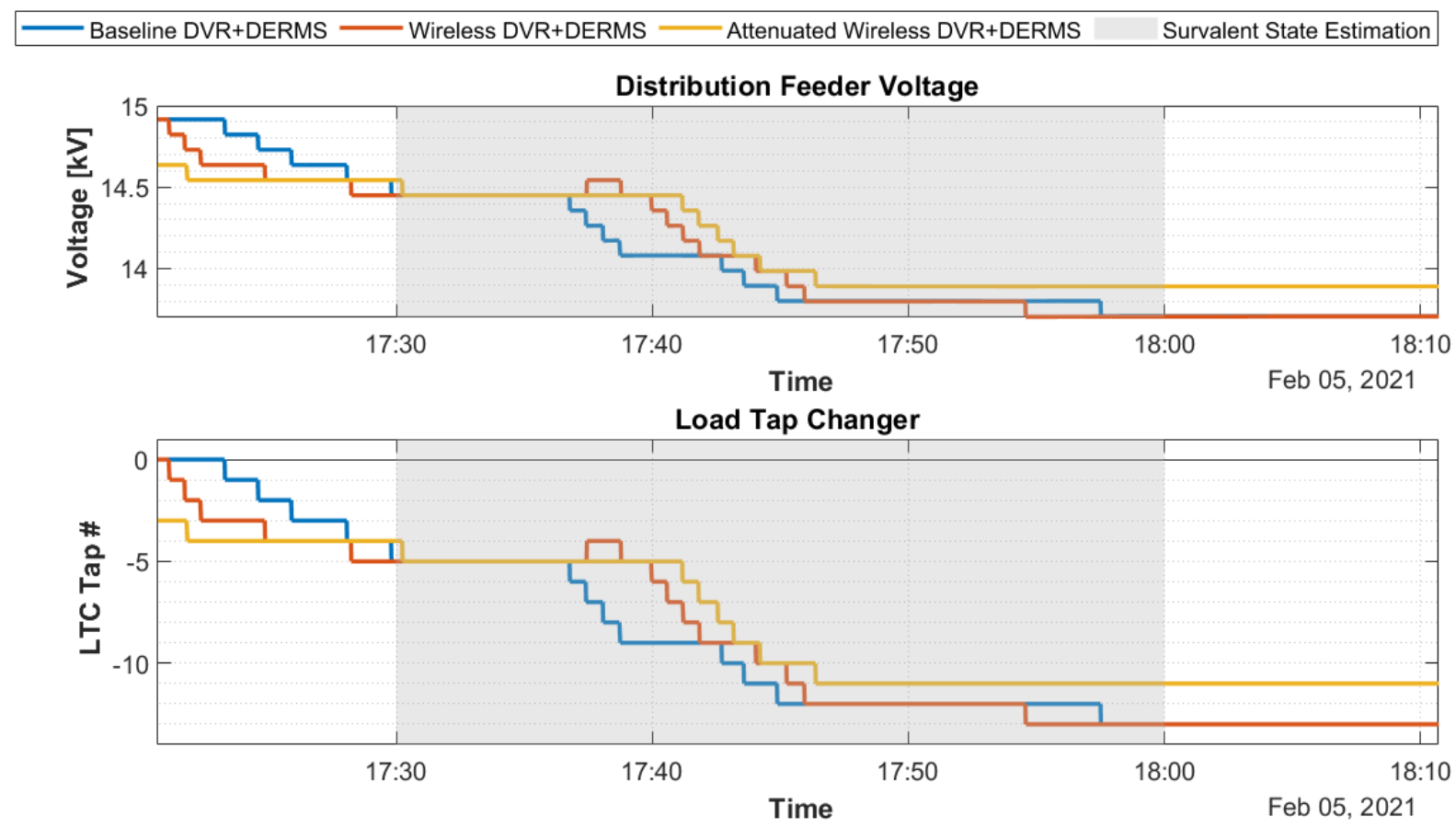

Figure ES-2. Experimental results for distribution feeder voltages based on changes in load tap changer set points using both dynamic voltage regulation and DERMS 


\section{Table of Contents}

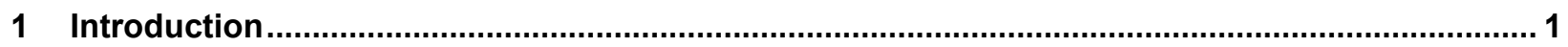

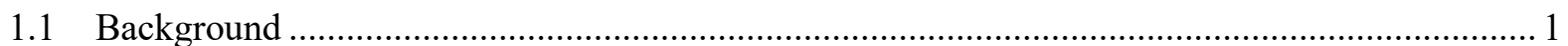

1.2 State-of-the-Art Wireless Distribution System Communication Methods..................................... 2

2 Evaluation of Wireless LTE Communications in a Direct Transfer Trip Relaying Scenario......... 4

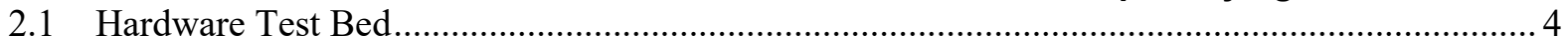

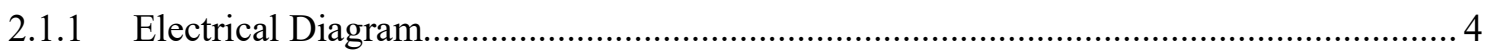

2.1.2 Communication Diagram ........................................................................ 5

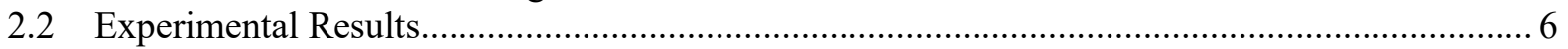

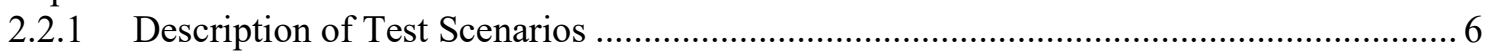

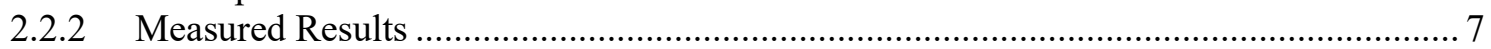

3 Validation and Measurement of Wireless LTE Communications in an Advanced Distribution

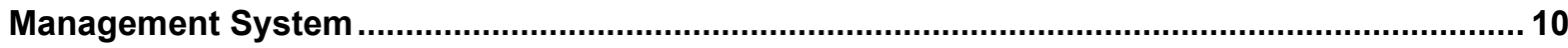

3.1 Background on Peak Load Management in Distribution Systems ........................................... 10

3.2 Motivation to Validate Private Wireless LTE for Grid Applications.......................................... 10

3.3 Overview of Advanced Distribution Management System Test Bed....................................... 10

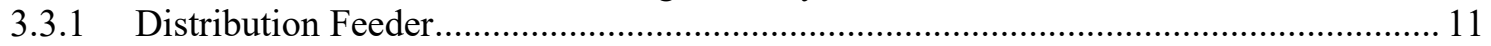

3.3.2 SurvalentONE Advanced Distribution Management System ..................................... 12

3.3.3 Distributed Energy Resource Management System Coordinator................................... 12

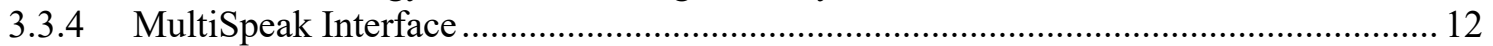

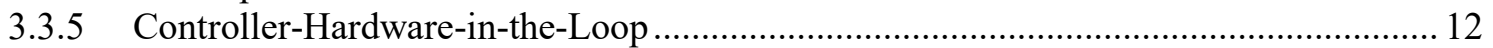

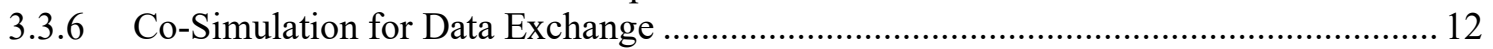

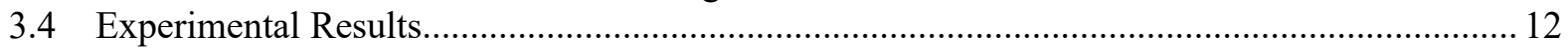

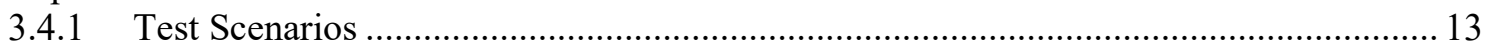

3.4.2 Measured Results and Discussion ............................................................... 13

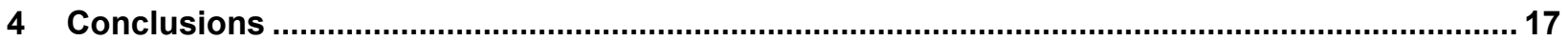

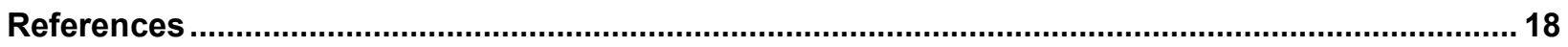

Appendix A. Validation of IEC 61850 Protocol Using Wireless Communications ........................... 20 


\section{List of Figures}

Figure ES-1. Box and whisker plots to show trends in latency calculations across all 70 DTT experiments (inclusive median). iv

Figure ES-2. Experimental results for distribution feeder voltages based on changes in load tap changer

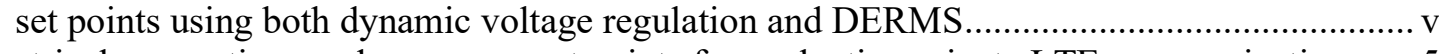

Figure 1. Electrical connections and measurement points for evaluating private LTE communications ..... 5

Figure 2. Network diagram for evaluating private LTE communications ............................................ 5

Figure 3. Images of laboratory experimental test bed for evaluating private LTE communications. Photos by NREL

Figure 4. Experimental measurements of line voltages and currents for the three-phase PV inverter when opening the ABB SACE E2.2S-A circuit breaker, with breaker trip command timing based on the SEL RTAC.

Figure 5. Box and whisker plots to show trends in latency calculations across all 70 DTT experiments

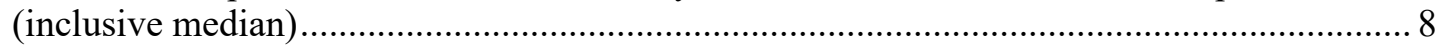

Figure 6. Bar plots to show trends in latency calculations across all 70 DTT experiments........................ 9

Figure 7. ADMS test bed experimental setup with all wired communications......................................... 11

Figure 8. ADMS test bed experimental setup using wireless LTE communications for LTC controller... 11

Figure 9. Experimental results for distribution feeder voltages based on change in LTC set point using DVR only

Figure 10. Experimental results for distribution feeder voltages based on change in LTC set point using both DVR and DERMS.

Figure A-1. Experimental setup for server-client communications with IEC 61850 communication protocol over wireless network Photos by NREL....

Figure A-2. Wireshark data capture of GOOSE messaging between IEC 61850 Client and IEC 61850

Server IEC over wireless network.................................................................................

software code to validate IEC 61850 communication protocol over wireless network. 21

\section{List of Tables}

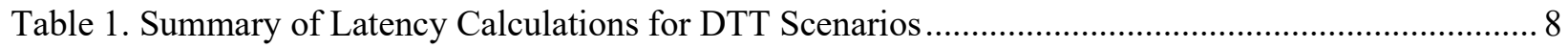

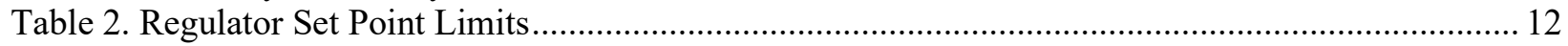

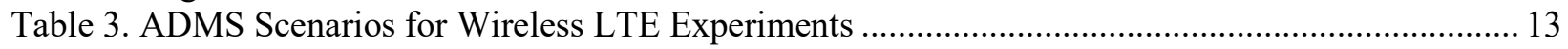

Table 4. Summary of Latency Calculations for ADMS Tests ............................................................... 15 


\section{Introduction}

\subsection{Background}

Increasing penetration levels of inverter-based distributed energy resources (DERs) in distribution systems are creating challenges in system operation. As demand for the seamless integration of DERs continues to increase, proper networking and communication systems are becoming important to support the DER infrastructure. As outlined by the U.S. Department of Energy's Multi-Year Project Plan for Grid Modernization, a modern grid should have increased resilience to all types of hazards, increased reliability, enhanced security, and superior flexibility to respond to variability, especially with a wider range of energy sources (Aalamifar and L. Lampe 2016). Controllers using both centralized and decentralized architectures can be used to achieve these goals (Achanta, Bradetich, and Fodero 2016). In both architectures, communications play a critical and integral role. Additionally, communication systems are critical in integrating DERs in microgrids. Communication systems enable microgrid controllers to control DERs and improve the resilience and reliability of microgrids (Chen 2010).

Currently, wired or proprietary wireless communications are used by advanced distribution management systems (ADMS) to detect the state of the system and to control the DERs for optimal operation. Wireless communications are an alternative solution that can enable connectivity between the dispersed assets used in distribution system applications. In Section 2 of this report, an experimental test bed is developed to enable the realistic evaluation of communication system characteristics for direct transfer trip (DTT) relaying of a photovoltaic (PV) inverter system using a private $900-\mathrm{MHz}$ spectrum wireless Long-Term Evolution (LTE) network. Private LTE network communications can be deployed to many remote DERs and other grid-edge devices to prevent costly infrastructure upgrades across distribution systems. In addition, this research provides a framework for evaluating the impact of signal strength and network traffic in communication systems. The results shown develop a better understanding of the best practices for using LTE communication systems across multiple utility applications, such as protective relaying, voltage regulation, and supervisory control and data acquisition (SCADA) system messages.

The use of communications enables a variety of grid modernization applications, such as protective functions, voltage regulation, advanced metering infrastructure (AMI), and SCADA. Power system utilities use diverse communication links - including dedicated fiber, Ethernet, telephone, cellular telecommunication networks, and radio frequency - to exchange information between various assets (Fix et al. 2019). In all of these applications, a robust and reliable infrastructure to enable efficient communications is required (Jawhar, Mohamed, and Al-Jaroodi 2017; Khattak, Mahmud, and Khan 2012; Kuzlu and Pipattanasomporn 2013). Multiple standards and protocols are used within these communication links to enable interoperability and cybersecurity.

Among the grid operation challenges that can benefit from communications, this research considers distribution system protection that focuses on systems with high penetrations of inverter-based DERs. The work presented here focuses particularly on the wireless communication infrastructure that is used in a DER protection relaying application. 
Many protection systems tend to operate using only local information and do not use communications for operation, but a few protection schemes use communications for normal operation (Maitra et al. 2017). DTT is one such protection scheme that uses communications to ensure distribution circuit-wide equipment protection. A DTT scheme is primarily used in transmission systems. Recently, these DTT schemes are being used by utilities to trip DERs offline whenever the substation breaker for a given distribution circuit opens (Namboodiri 2013). DTT relaying requires low-latency communications, and utilities often use fiber or dedicated lease lines to implement DTT. With the increased deployment of DERs, the cost of providing fiber-based communications to all DERs is prohibitive, and leased lines are being phased out by the industry, necessitating alternative but effective communication methods. A wireless network solution appears ideal to inexpensively connect many DERs that are spread out over a relatively large area if DTT latency can be decreased to an acceptable level.

In this report, a private broadband wireless network solution is evaluated to implement a realistic communication network for distribution system applications relevant to U.S. utility companies. The objective of this work is to demonstrate a hardware test bed for evaluating communication performance in protection-focused scenarios (e.g., DTT of a circuit breaker). This paper presents the results from experiments evaluating communication latency in a DTT scenario.

\subsection{State-of-the-Art Wireless Distribution System Communication Methods}

From a broader perspective, communications can be obtained through a wired physical connection (copper, fiber optics) or wirelessly (Wi-Fi, ZigBee, WiMAX, or cellular). Multiple works in the literature discuss the use of physical connections (copper, fiber optics) for communications in distribution systems (Obaidat, Anpalagan, and Woungang 2012). Here, we focus on the use of wireless communications in distribution systems. This section describes the different network classifications and various wireless technologies that can be used in them to enable smart grid applications in distribution systems. The wireless technologies presented in this section can be used toward creating three types of networks that enable data exchange between the management systems and devices in the electric grid (Pacheco, Tunc, and Hariri 2016):

- Home-area network (HAN): Residential control centers or consumers use HANs to monitor and control smart home devices. Typically, the required coverage for such networks is a few thousand square feet, and they support fewer than tens of kbps.

- Neighbor-area network (NAN): NANs act as a relay between a group of smart meters or devices in a neighborhood and a WAN.

- Wide-area network (WAN): WANs typically aggregate data from a wide array of devices and move them to utility control centers and vice versa. WANs cover a large area and support high-bandwidth data transmission.

The following wireless technologies are used in the different area networks to enable data transmission between the end devices and the utility control centers:

- ZigBee: ZigBee is one protocol used in HAN. It is built on top of the standard built by the Institute of Electrical and Electronics Engineers (IEEE) (IEEE 802.15.4). ZigBee has 
been proposed in the literature for use in monitoring grid-connected PV systems (Schneider 2019) and in smart metering applications (Shariff, Rahim, and Hew 2015).

- Wi-Fi: Wi-Fi is a prominent and mature technology that has been used in HANs to support smart grid applications. In recent years, Wi-Fi technology has been used in a wide range of devices in HANs for sensing and control applications.

- WiMAX: Worldwide Interoperability for Microwave Access (WiMAX) is another technology that has been proposed for use in NANs and WANs to enable smart grid applications (Simms and Johnson 2015). WiMAX can be used in NANs to collect smart meter traffic from a neighborhood of smart meter devices.

- Cellular communications: Cellular communications is the transmission of data via landbased cellular towers. Wireless LTE networks have become attractive communications methods for utility companies because of their flexibility and interoperability (DOE 2015). Because the reach of such networks is large, they are primarily used in WAN data transmission. At present, there are four generations of cellular technologies, and work is being done to implement the fifth generation $(5 \mathrm{G})$ around the world. The maturity of this technology and the continuous support for growth toward 5G makes this technology a viable solution for the future communications needs of smart grid applications.

These cellular technologies use 850-, 900-, 1,800-, and 1,900-MHz frequency bands (Whitehead and Smith 2008). The wireless technology used in this research is a private $900-\mathrm{MHz}$ spectrum allocated for wireless LTE. The 900-MHz spectrum network used in this research is licensed for experimental work. 


\section{Evaluation of Wireless LTE Communications in a Direct Transfer Trip Relaying Scenario}

To evaluate LTE wireless communication latency, a DTT of a PV system is selected as an example of a smart grid scenario. DTT is a protection function of distribution systems that quickly disconnects or shuts down distributed generation if the substation breaker for a given distribution circuit opens (IEEE 2003). The objective is to avoid any chance of islanding the distribution circuit and all connected customer equipment and mitigating any potential loadrejection overvoltage concerns stemming from installed DERs. DTT is a representative protection function that likely requires the highest level of communication priority and results in extremely low latency, even under congested communication channel conditions. This is effectively a test of the highest performance application for an ADMS-plus-communication system and is an application for which utilities need a dependable wireless communication solution. DTT requires low latency, but it also has low-bandwidth applications analogous to many other system protection-related functions potentially implemented either directly or via ADMS integration. DTT requires communications to multiple remote points on a distribution system (i.e., the sites of all or many DERs), all with low latency. Zawada (2000) provided details on a pilot project to send DTT signals between DERs and the circuit breakers over a cellular network using the International Electrotechnical Commission (IEC) 61850 protocol. This paper discussed the advantages of using wireless communications for DTT systems; however, Zawada (2000) did not provide a statistical analysis of the delays under various communication loads and other important characteristics of the communications. This is crucial for the future implementation of wireless communications for DTT systems.

\subsection{Hardware Test Bed}

\subsubsection{Electrical Diagram}

The hardware test bed is illustrated in Figure 1. Electrically, a 100-kW PV inverter is operated from a $250-\mathrm{kW}$ rated PV simulator and connected to an AC grid simulator. The system is instrumented to collect the three-phase line voltage and line current waveforms on the grid simulator side of the circuit breaker shown in Figure 1, which isolates the DER from the distribution grid. The Schweitzer Engineering Laboratories (SEL) real-time automation controller (RTAC) is configured to send (on user command) a "trip" signal to the circuit breaker (to break under load) via Modbus/Transmission Control Protocol (TCP) protocol. This command is passed through the $4 \mathrm{G} \mathrm{LTE}$ wireless system, and communication latency measurements are obtained by the oscilloscope, as shown in Figure 1. This setup can be modified to evaluate the impact of $5 \mathrm{G}$ communications on the control and protection of DERs as well. 


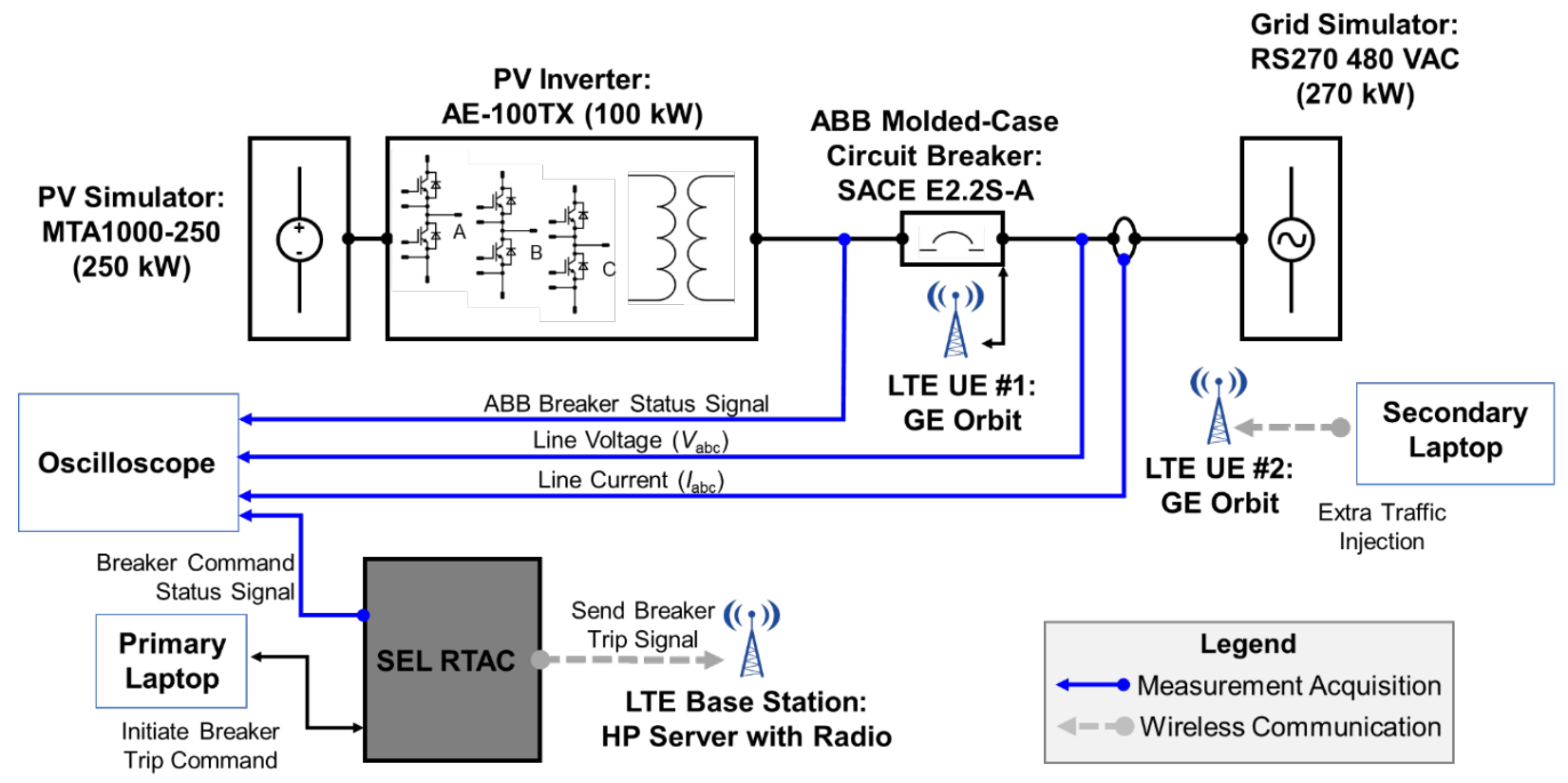

Figure 1. Electrical connections and measurement points for evaluating private LTE communications

\subsubsection{Communication Diagram}

The communication system uses off-the-shelf, 4G LTE hardware, including one "base station" (shown in Figure 1) that consists of an HP server with an LTE radio card installed and two GE Orbit access points. The network diagram in Figure 2 shows the two access points. These access points operate in the $900-\mathrm{MHz}$ frequency band. A temporary experimental license was obtained to use these devices for the research in this work because the use of the frequency bands on which these operate is not currently allocated for LTE service.

Three separate virtual local area networks are used to segregate traffic based on the priority of their messages. Prioritization is possible because the implemented 4G LTE system is a private licensed system, not a public system in which all traffic has the same general priority. The three networks are for the following types of messages: AMI, SCADA, and DTT/protection-related traffic. For example, DTT traffic would typically be set to have a higher priority than AMI traffic.

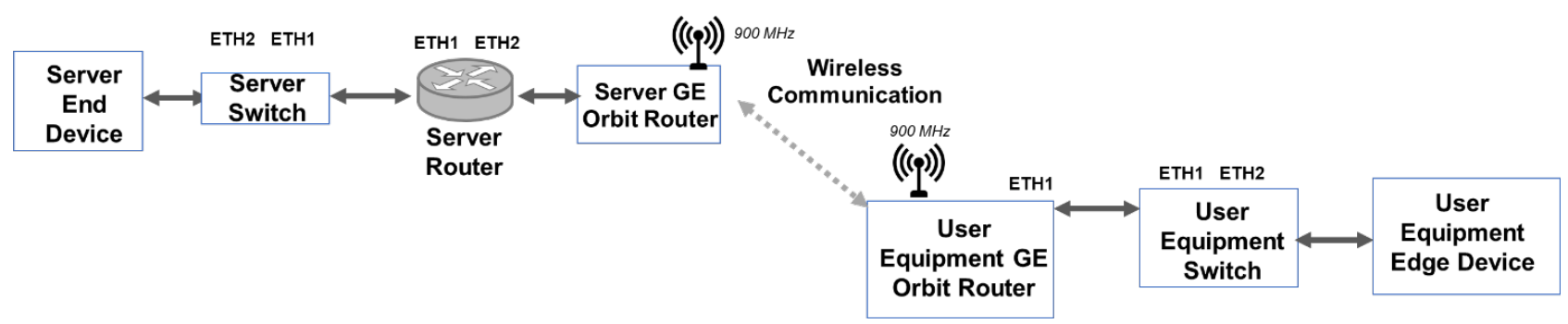

Figure 2. Network diagram for evaluating private LTE communications 


\subsection{Experimental Results}

With the experimental test bed illustrated in Figure 1, a DTT command of the PV inverter is sent via the private LTE network, as shown in Figure 2. Figure 3 shows images of the experimental test bed in the laboratory setting.
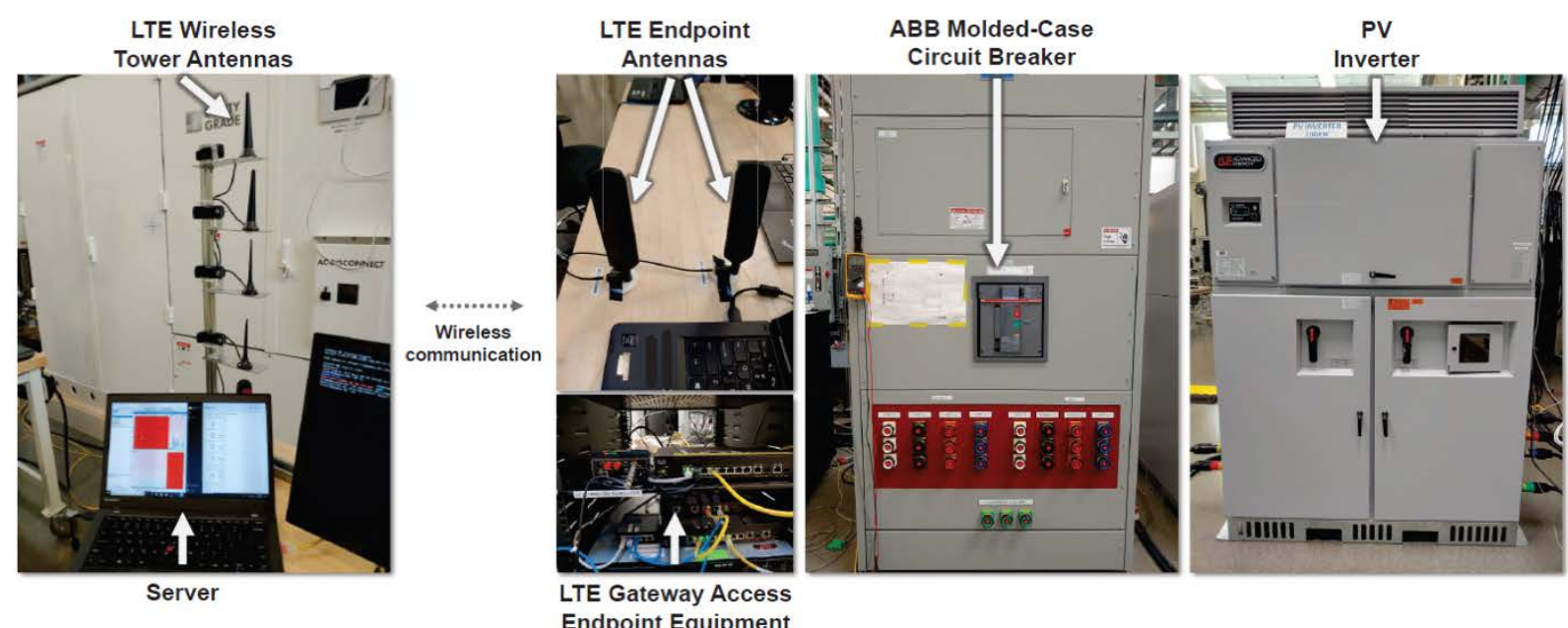

Endpoint Equipment

Figure 3. Images of laboratory experimental test bed for evaluating private LTE communications. Photos by NREL

\subsubsection{Description of Test Scenarios}

\subsubsection{Wired Ethernet Connection}

In this test, the wireless network shown in Figure 2 was not used, and the "send breaker trip signal" line shown in Figure 1 was simply connected from the SEL RTAC directly to the breaker with an Ethernet cable. There was no "extra traffic injection" via the secondary laptop shown in Figure 1. This case effectively set the minimum latency possible for a DTT in the implemented test bed.

\subsubsection{Message Prioritization}

As noted in the previous section, message prioritization is possible with the private licensed $4 \mathrm{G}$ LTE system implemented for experiments discussed in this paper. The benefit of message prioritization with a private LTE network is to reduce the latency of time-critical messages when the network becomes congested with other traffic. The private LTE wireless system can use a priority queuing lane for breaker traffic higher than any other network traffic.

Without extra network traffic being injected by the second laptop shown in Figure 1 in this setup, the only messages being transmitted are the breaker communication messages for DTT. If extra network traffic is being injected by the second laptop shown in Figure 1, however, message prioritization is achieved by setting the breaker communications and network traffic on two separate network priorities.

When the breaker communications are set to a higher priority, the extra injected network traffic is on a lower priority path, prioritizing the communications from the SEL RTAC to the breaker shown in Figure 1. The network traffic can also be set to a higher priority than the breaker 
communications, which leads to a higher latency in timing from the SEL RTAC to the breaker. It is expected that the DTT timing will be shorter for scenarios when the breaker communications are on a higher priority path than when having the network traffic on a higher priority path.

\subsubsection{Signal Attenuation}

If a wireless LTE system were to be deployed to support the DTT functionality of a PV inverter system, the wireless transmitting tower would typically be built on leased land or on a utilityowned asset location. To emulate a weaker signal caused by propagation characteristics in a given LTE coverage area, attenuators were placed at the breaker communication signal's LTE gateway access point. Throughout the results in this paper, "normal wireless" refers to a strong LTE signal in a given LTE coverage area, and "weak wireless" refers to an attenuated LTE signal that might be realized farther from the wireless tower in a real-world power system scenario (e.g., the DER location is near the cell edge). The "weak" wireless signal is approximately $20 \mathrm{~dB}$ lower in strength than the "normal" wireless signal. The received signal strength indication (RSSI) is a metric that indicates the strength of the signal received. In this report, the normal wireless signal had RSSI values between $-70 \mathrm{~dB}$ and $-80 \mathrm{~dB}$, and the weak wireless signal had RSSI values between $-90 \mathrm{~dB}$ and $-100 \mathrm{~dB}$.

\subsubsection{Measured Results}

An example of measurements from one DTT event (wireless communications with no load traffic) is shown in Figure 4.

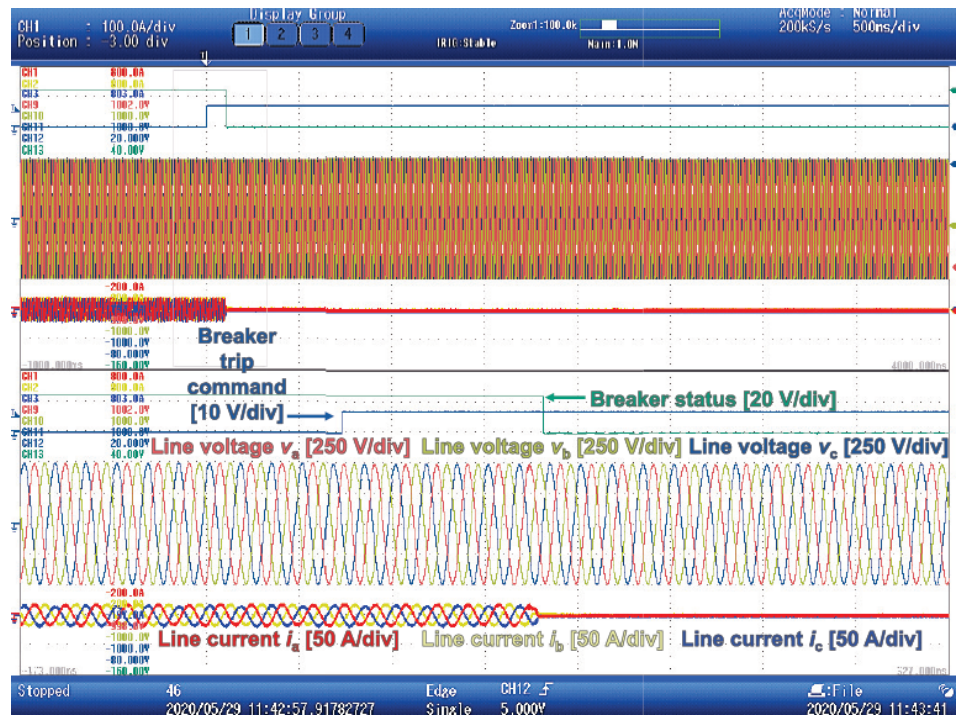

Figure 4. Experimental measurements of line voltages and currents for the three-phase PV inverter when opening the ABB SACE E2.2S-A circuit breaker, with breaker trip command timing based on the SEL RTAC

A method to measure the latency between the sent trip command and the completion of the DTT event is from measuring the timing differences between the analog output signal from the SEL RTAC (which is enabled as soon as the trip command is sent) and when the PV inverter's threephase output current, $I_{\mathrm{abc}}$, downstream of the breaker drops to zero. The different communication scenarios were repeated experimentally 10 times each to provide a statistically relevant understanding of the results. 
Figure 5 shows the box and whisker plot representation for 70 DTT experiments (10 tests per scenario), with respective data tabulated in Table 1. As summarized in Table 1, the wired Ethernet connection has the shortest communication timing: $59.6879 \mathrm{~ms}$ from initial breaker trip command to zero output current. This is expected because a direct connection will have the advantage of "single hop" latency.

Table 1. Summary of Latency Calculations for DTT Scenarios

\begin{tabular}{|c|c|c|c|c|c|}
\hline Scenario & Test Description & $\begin{array}{l}\text { Average } \\
\text { Time to } \\
\text { Trip (ms) }\end{array}$ & $\begin{array}{l}\text { Maximum } \\
\text { Time to } \\
\text { Trip (ms) }\end{array}$ & $\begin{array}{l}\text { Minimum } \\
\text { Time to } \\
\text { Trip (ms) }\end{array}$ & $\begin{array}{c}\text { Range in } \\
\text { Timing } \\
\text { (ms) }\end{array}$ \\
\hline 1 & Wired Ethernet connection & 59.69 & 88.87 & 46.19 & 42.68 \\
\hline II & Normal wireless, no network traffic & 74.01 & 120.08 & 55.09 & 64.99 \\
\hline III & $\begin{array}{l}\text { Normal wireless, high-priority } \\
\text { breaker communications }\end{array}$ & 80.16 & 122.77 & 53.59 & 69.19 \\
\hline IV & $\begin{array}{l}\text { Normal wireless, low-priority } \\
\text { breaker communications }\end{array}$ & 804.08 & 978.93 & 569.13 & 409.80 \\
\hline $\mathrm{V}$ & $\begin{array}{c}\text { Weak (attenuated) wireless, no } \\
\text { network traffic }\end{array}$ & 105.61 & 181.49 & 56.04 & 125.45 \\
\hline VI & $\begin{array}{l}\text { Weak (attenuated) wireless, high- } \\
\text { priority breaker communications }\end{array}$ & 95.50 & 160.72 & 56.89 & 103.83 \\
\hline VII & $\begin{array}{l}\text { Weak (attenuated) wireless, low- } \\
\text { priority breaker communications }\end{array}$ & 1056.09 & 1919.21 & 524.54 & 1394.68 \\
\hline
\end{tabular}

\section{Box and Whisker Plots of Latency Calculations for Direct Transfer Trip Experiments}

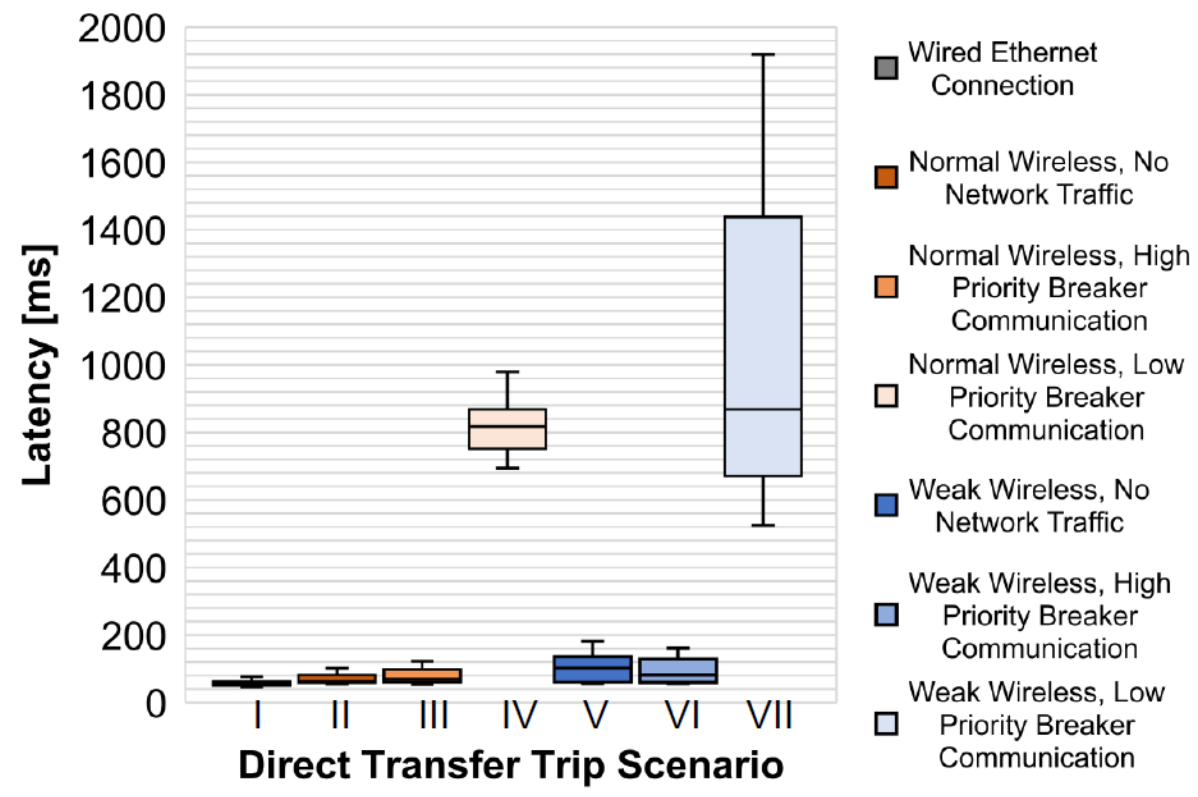

Figure 5. Box and whisker plots to show trends in latency calculations across all 70 DTT experiments (inclusive median) 
As illustrated in Figure 5, the scenarios with lower priority for breaker communications (higher priority for network traffic) resulted in the wider ranges in timing calculations and the highest average latencies for the breaker to trip. For the high-priority breaker communication scenarios, the "weak wireless" (scenario VI) had a $40 \%$ greater range in timing than the "normal wireless" (scenario III). Similarly, for the low-priority breaker communication scenarios, the "weak wireless" (scenario VII) had a 109\% greater range in timing than the "normal wireless" (scenario IV). For scenario VII, the network traffic had a higher message prioritization, and the breaker communication signal was also attenuated. This resulted in a much wider range in trip timing $(1394.68 \mathrm{~ms})$ than the "normal wireless," low-priority breaker communication timing range $(409.80 \mathrm{~ms})$. The latency calculation data summarized in Table 1 are also illustrated as a group of bar charts in Figure 6.

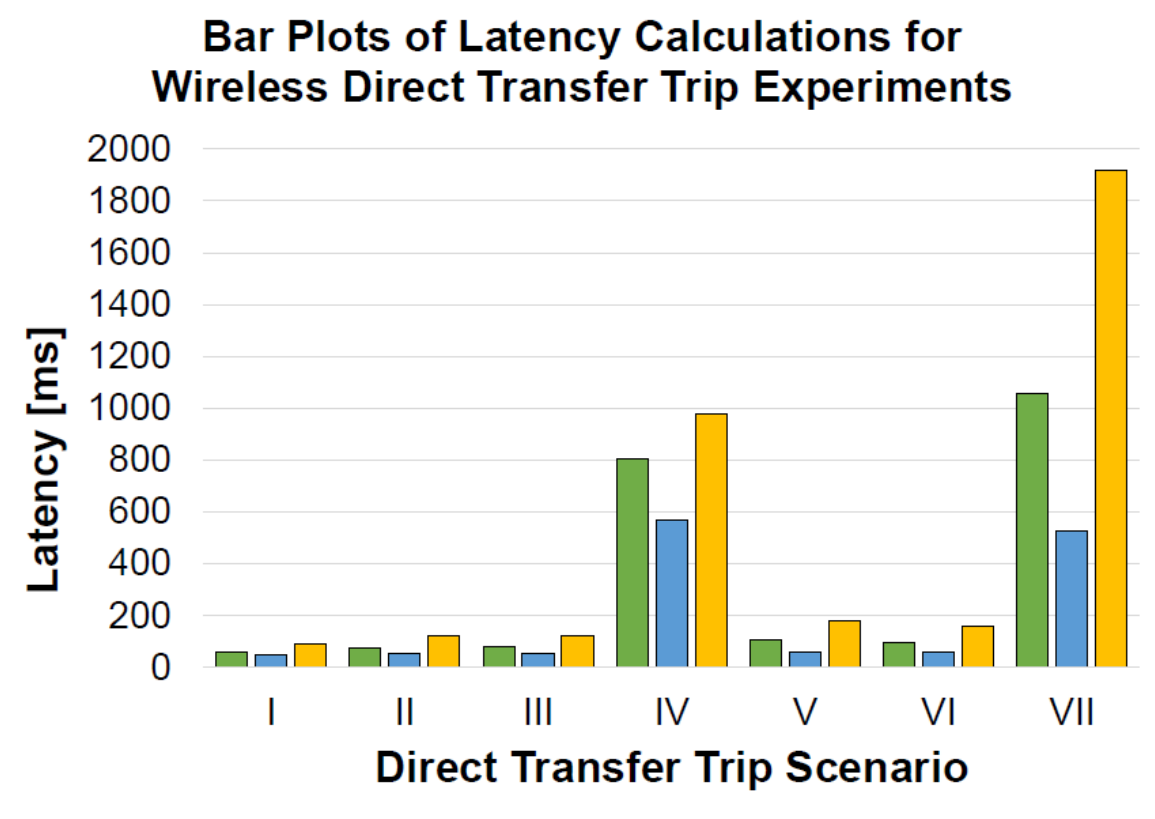

$\square$ Average Time until Trip $\quad \square$ Minimum Time until Trip $\quad \square$ Maximum Time until Trip

Figure 6. Bar plots to show trends in latency calculations across all 70 DTT experiments 


\section{Validation and Measurement of Wireless LTE Communications in an Advanced Distribution Management System}

\subsection{Background on Peak Load Management in Distribution Systems}

The purpose of distribution systems is to deliver power based on the load demand from customers. During peak load demand periods, utilities face challenges to maintain the performance aspects of their distribution systems, such as reliability, cost, and power quality. To help mitigate the impacts from peak demand periods, peak load management (PLM) is a way to optimize the operation of distribution systems to perform with increased efficiency and to reduce the overall cost for utilities during such periods. The National Renewable Energy Laboratory (NREL) developed a test bed to study different ADMS scenarios in real time. The test bed application used in this report is specifically for showcasing PLM performance at a distribution feeder. The motivation for studying wireless communications within an ADMS is to demonstrate two-way communication performance from a grid system level.

\subsection{Motivation to Validate Private Wireless LTE for Grid Applications}

Utilities are increasingly looking to privately owned LTE networks to support their grid modernization efforts, providing secure, low-latency wireless connectivity to the thousands of remote sensors and other smart devices installed throughout the modern grid. Examples include AMI; volt/volt ampere reactive controls; fault location, isolation, and service restoration systems; DER integration; and other use cases necessary for utilities to provide safe, reliable, and costefficient energy to their customers. Private wireless LTE communication systems offer economic advantages to utility ratepayers over other solutions such as direct fiber or microwave.

LTE is the state-of-the-art standard from the worldwide $3^{\text {rd }}$ Generation Partnership Project standards body. LTE's key characteristics include its advanced cybersecurity features, an existing worldwide device ecosystem, and years of worldwide operator experience, making it a mature, reliable technology on the evolutionary path to 5G. As the most-deployed advanced cellular technology in the United States, a private LTE network will provide a utility interoperability and ease of roaming with other LTE systems.

Section 3 of this report demonstrates the application of private wireless LTE in a two-way communication application using an ADMS. This provides insight into the impacts of using wireless on grid performance (specifically on PLM performance in the chosen ADMS use case in this work).

\subsection{Overview of Advanced Distribution Management System Test Bed}

Diagrams of the ADMS test bed are shown for both the wired configuration and wireless configuration of the setup in Figure 7 and Figure 8, respectively. 


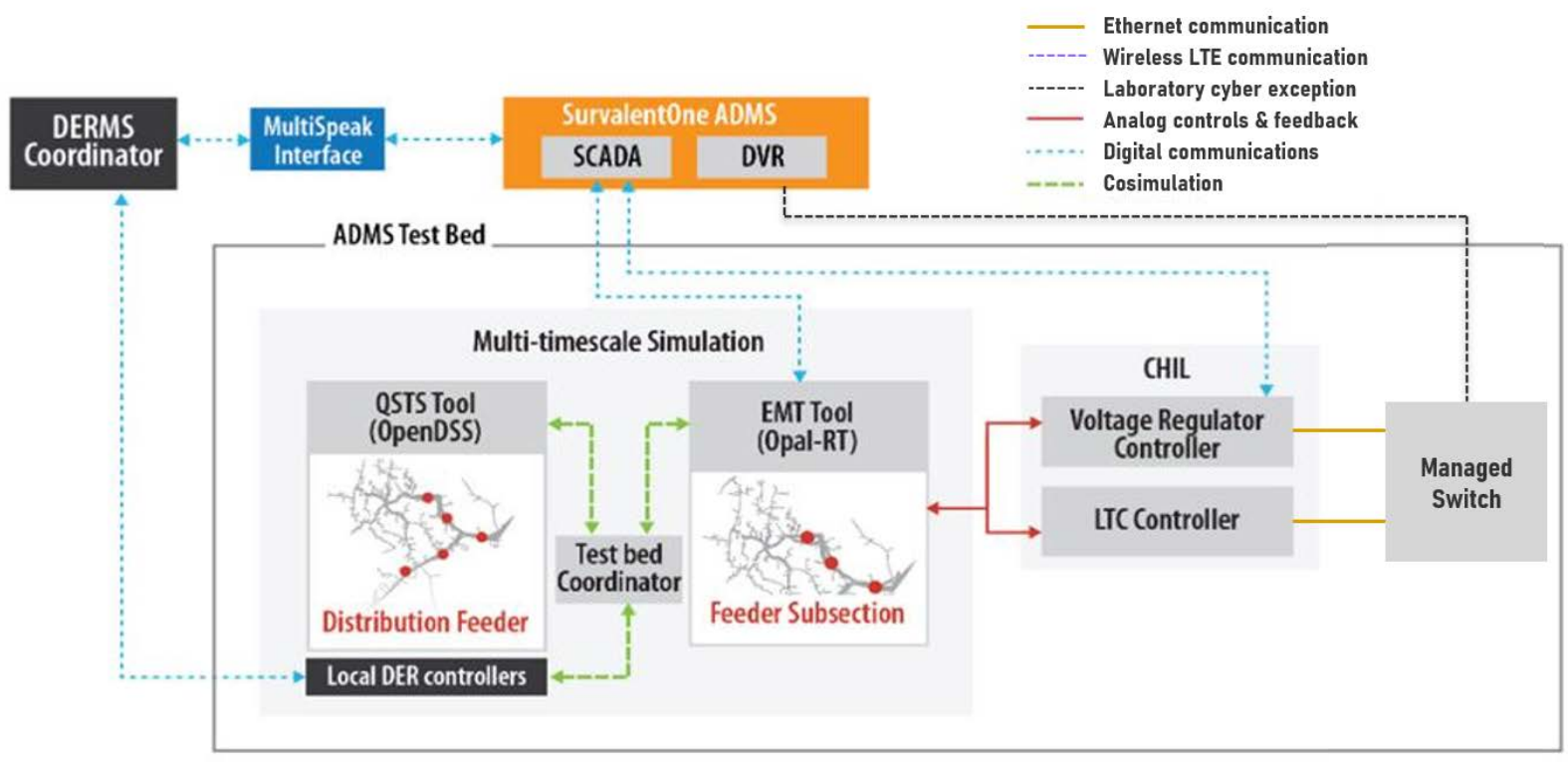

Figure 7. ADMS test bed experimental setup with all wired communications

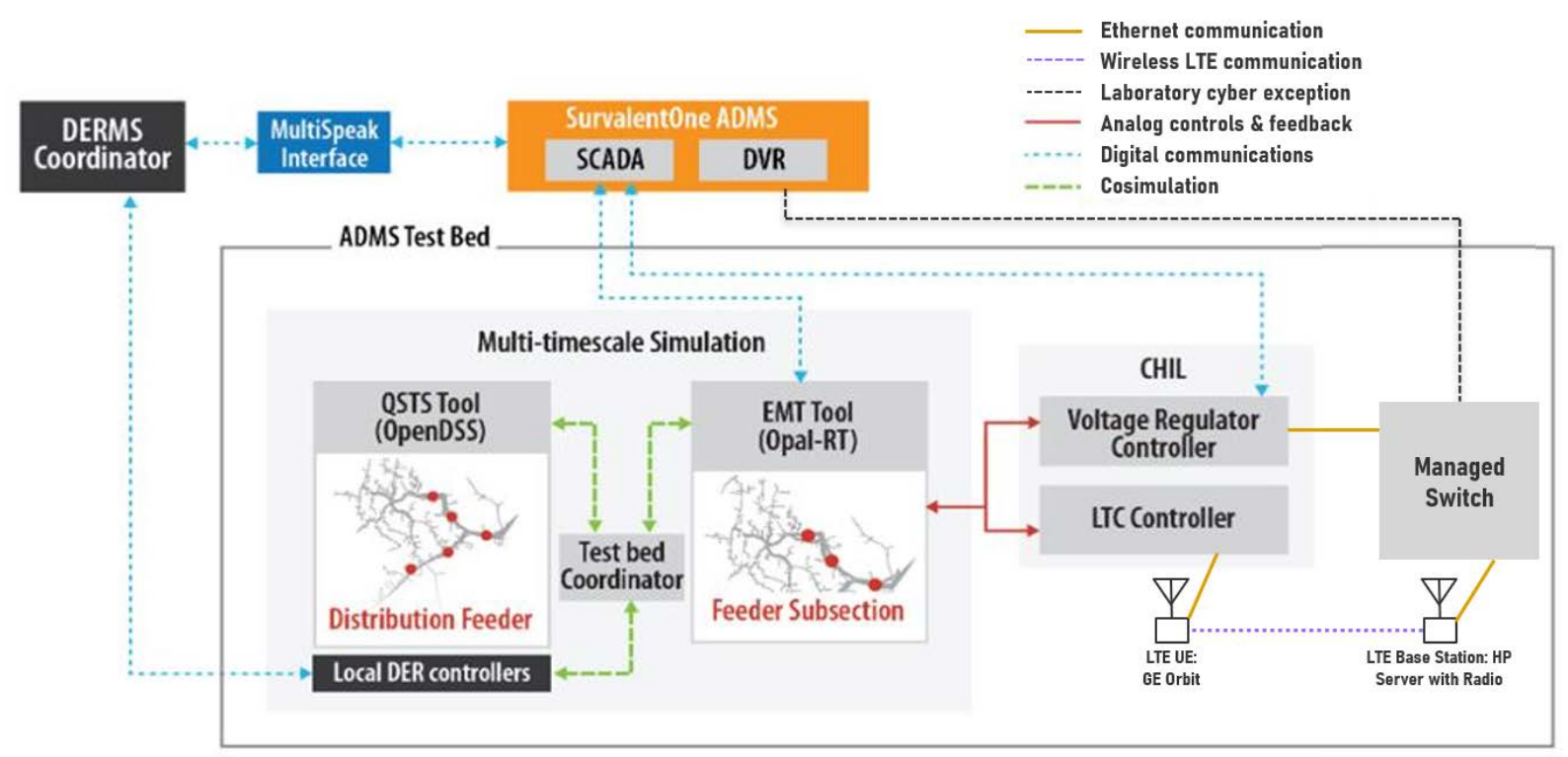

Figure 8. ADMS test bed experimental setup using wireless LTE communications for LTC controller

\subsubsection{Distribution Feeder}

In this ADMS test bed, the distribution feeder model is developed based on data from Holy Cross Energy. Most of the utility feeder is simulated in OpenDSS, which is a quasi-static timeseries simulator, whereas a subsection of the feeder is simulated via an OPAL-RT digital realtime simulator (Padullaparti et al. 2021). More details on the distribution feeder model can be found in Padullaparti et al. (2021). 


\subsubsection{SurvalentONE Advanced Distribution Management System}

The SurvalentONE ADMS is a Windows-based platform with a fully integrated SCADA, outage management system, and distribution management system (Survalent 2020). For the ADMS test bed setup in this work, the dynamic voltage regulation (DVR) application is used to regulate system voltages by providing set points to a load tap changer (LTC) and a voltage regulator. The nominal feeder voltage is $8.31 \mathrm{kV}$ line-to-neutral ( $14.4 \mathrm{kV}$ line-to-line), and the corresponding nominal voltages in the secondary is $120 \mathrm{~V}$ line-to-neutral. The set point limits are included in Table 2 (secondary voltage values; the actual feeder voltage is $8.31 \mathrm{kV}$, which translates to a nominal secondary voltage of $120 \mathrm{~V}$ ).

Table 2. Regulator Set Point Limits

\begin{tabular}{cccccc}
\hline Regulator & $\begin{array}{c}\text { Initial Set } \\
\text { Point }\end{array}$ & $\begin{array}{c}\text { Near High } \\
\text { Limit }\end{array}$ & $\begin{array}{c}\text { Near Low } \\
\text { Limit }\end{array}$ & $\begin{array}{c}\text { Far High } \\
\text { Limit }\end{array}$ & $\begin{array}{c}\text { Far Low } \\
\text { Limit }\end{array}$ \\
\hline Load tap changer & $121 \mathrm{~V}$ & $124 \mathrm{~V}$ & $115 \mathrm{~V}$ & $124 \mathrm{~V}$ & $114 \mathrm{~V}$ \\
Voltage regulator & $123 \mathrm{~V}$ & $124 \mathrm{~V}$ & $115 \mathrm{~V}$ & $124 \mathrm{~V}$ & $114 \mathrm{~V}$ \\
\hline
\end{tabular}

Source: Padullaparti et al. (2021)

\subsubsection{Distributed Energy Resource Management System Coordinator}

In addition to DVR, a distributed energy resource management system (DERMS) is also available within the ADMS (based on real-time optimal power flow) to control distributed battery energy storage systems to further reduce feeder power (Padullaparti et al. 2021). For PLM, the DERMS aims to track a power target for each phase set by the ADMS. For voltage regulation, the DERMS is used to keep system voltages within user-defined bounds (Padullaparti et al. 2021).

\subsubsection{MultiSpeak Interface}

MultiSpeak is a communication standard that enables coordinated operations between the ADMS and the DERMS (Zawada 2000). To enable or disable the DERMS, the ADMS sends MultiSpeak messages for both PLM and voltage regulation (Padullaparti et al. 2021).

\subsubsection{Controller-Hardware-in-the-Loop}

To operate the test bed as a controller-hardware-in-the-loop setup, the eMEGASIM platform from OPAL-RT interfaces with the LTC and voltage regulator hardware controllers. More details on the controller-hardware-in-the-loop setup are described in Padullaparti et al. (2021).

\subsubsection{Co-Simulation for Data Exchange}

The co-simulation platform was developed using Python to coordinate data exchanges among the different subsystems within the ADMS test bed. Specifically, this platform streams required data from OpenDSS to Opal-RT, receives commands for the LTC and voltage regulator controllers from the CHIL via OPAL-RT, receives set points from local DERMS controllers, and implements set points in the feeder model (Padullaparti et al. 2021).

\subsection{Experimental Results}

This section presents the different test scenarios ran using the experimental setup and a discussion on the results. 


\subsubsection{Test Scenarios}

In this work, a subset of the ADMS test scenarios presented in Padullaparti et al. (2021) was run using the test bed.

\subsubsection{Advanced Distribution Management System Scenarios}

In Padullaparti et al. (2021), four different ADMS scenarios was shown. In this work, two of the four tests from Padullaparti et al. (2021) was used. These ADMS scenarios are listed in Table 3 and described next.

Table 3. ADMS Scenarios for Wireless LTE Experiments

\begin{tabular}{cccc}
\hline Scenario & ADMS DVR & DERMS PLM & $\begin{array}{c}\text { DERMS } \\
\text { Voltage } \\
\text { Regulator }\end{array}$ \\
\hline DVR only & Enabled & Disabled & Disabled \\
DVR and DERMS & Enabled & Enabled & Enabled \\
\hline
\end{tabular}

Source: Padullaparti et al. (2021)

\subsection{Dynamic Voltage Regulation Only}

DVR is used by the ADMS in this experiment to reduce peak demand by reducing system voltages (Padullaparti et al. 2021). This application within the ADMS uses feedback from voltage measurements from selected locations across the distribution feeder. In this scenario, there is no action requested from the DERMS. After initiating each test, the ADMS DVR is enabled after 15 minutes of operation.

\subsection{Dynamic Voltage Regulation and Distributed Energy Resource Management System}

The coordinated operation of both the ADMS DVR and DERMS is tested. After initiating each test, the DERMS voltage regulation, DERMS PLM, and ADMS DVR are enabled after 15 minutes of operation.

\subsubsection{Wireless Scenarios}

In this work, the private LTE wireless network was set up to communicate between the LTC and a managed switch that configures communications to the ADMS. The three different communication scenarios tested in this phase from the LTC included wired (baseline) Ethernet, wireless without attenuation, and attenuated wireless communications. The wireless network in this application follows the same configuration shown in the network diagram in Figure 2.

\subsubsection{Measured Results and Discussion}

The controller-hardware-in-the-loop experiment starts with the distribution feeder voltage at $14.916 \mathrm{kV}$ line-to-line (equivalent secondary line-to-neutral voltage $124.3 \mathrm{~V}$ ) and an initial set point of $14.52 \mathrm{kV}$ (equivalent secondary line-to-neutral voltage $121 \mathrm{~V}$ ). During the first 15 minutes, the system settles to the $14.52-\mathrm{kV}$ (equivalent secondary line-to-neutral voltage $121 \mathrm{~V}$ ) initial set point. At this point, either DVR only or DVR and DERMS are enabled. This is where Survalent performs the state estimation and sends a set point command each minute until Survalent settles at its lowest limit. It can be observed that there are some slight fluctuations in 
the tap controller response caused by subsequent changes in the system following this initial response.

Figure 9 and Figure 10 show the experimental results for the different ADMS and wireless scenarios mentioned. Table 4 includes the settling times for all six experiments in this work. For all experiments, the time resolution is 2 seconds, which is based on how the system updates the set points every 2 seconds. The settling time metric in Table 4 is based on the times when the system settles to its initial set point to when the tap controller settles to its final value. As shown in Figure 9 and Figure 10, the initial set point is reached for all tests at approximately 17:30.

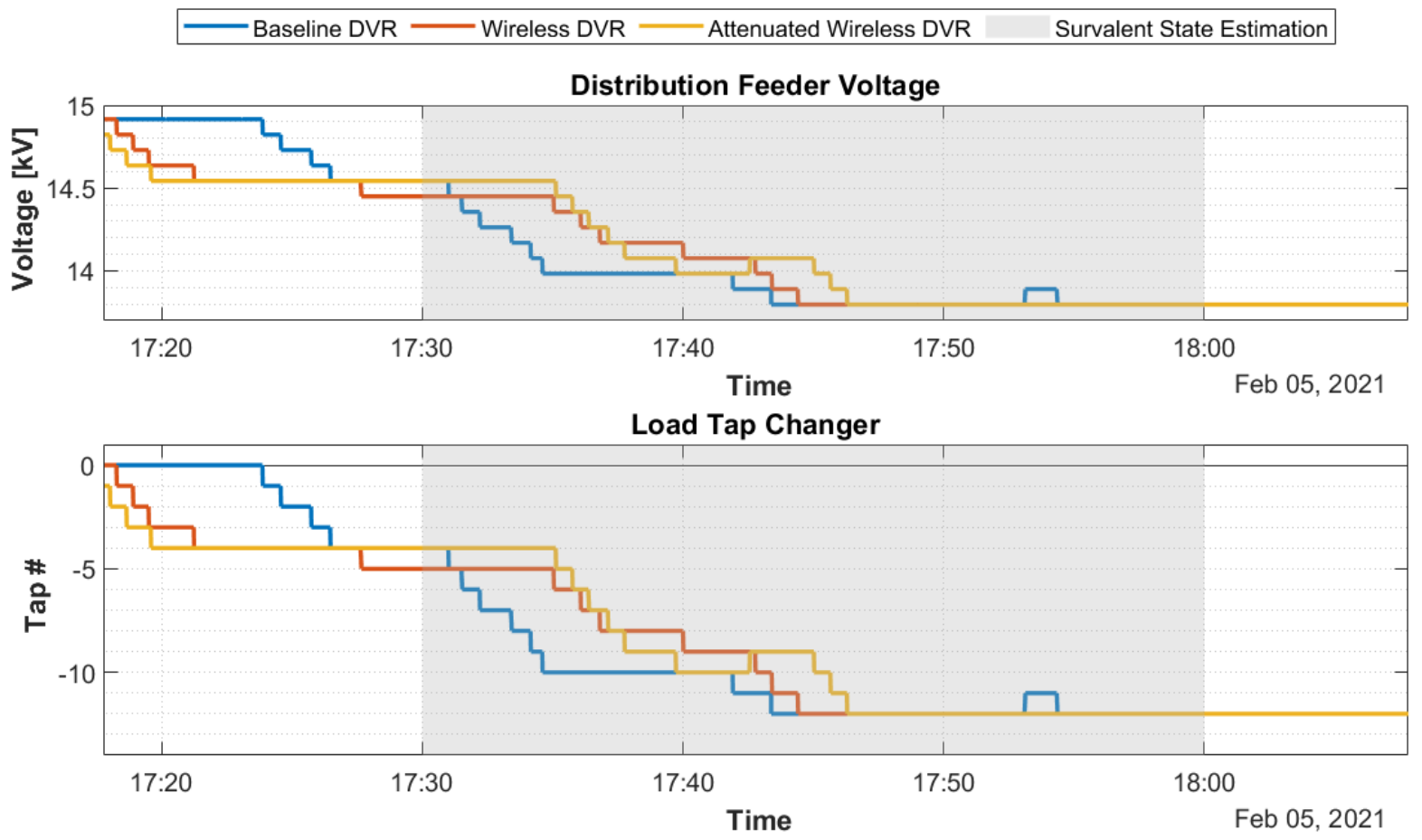

Figure 9. Experimental results for distribution feeder voltages based on change in LTC set point using DVR only 

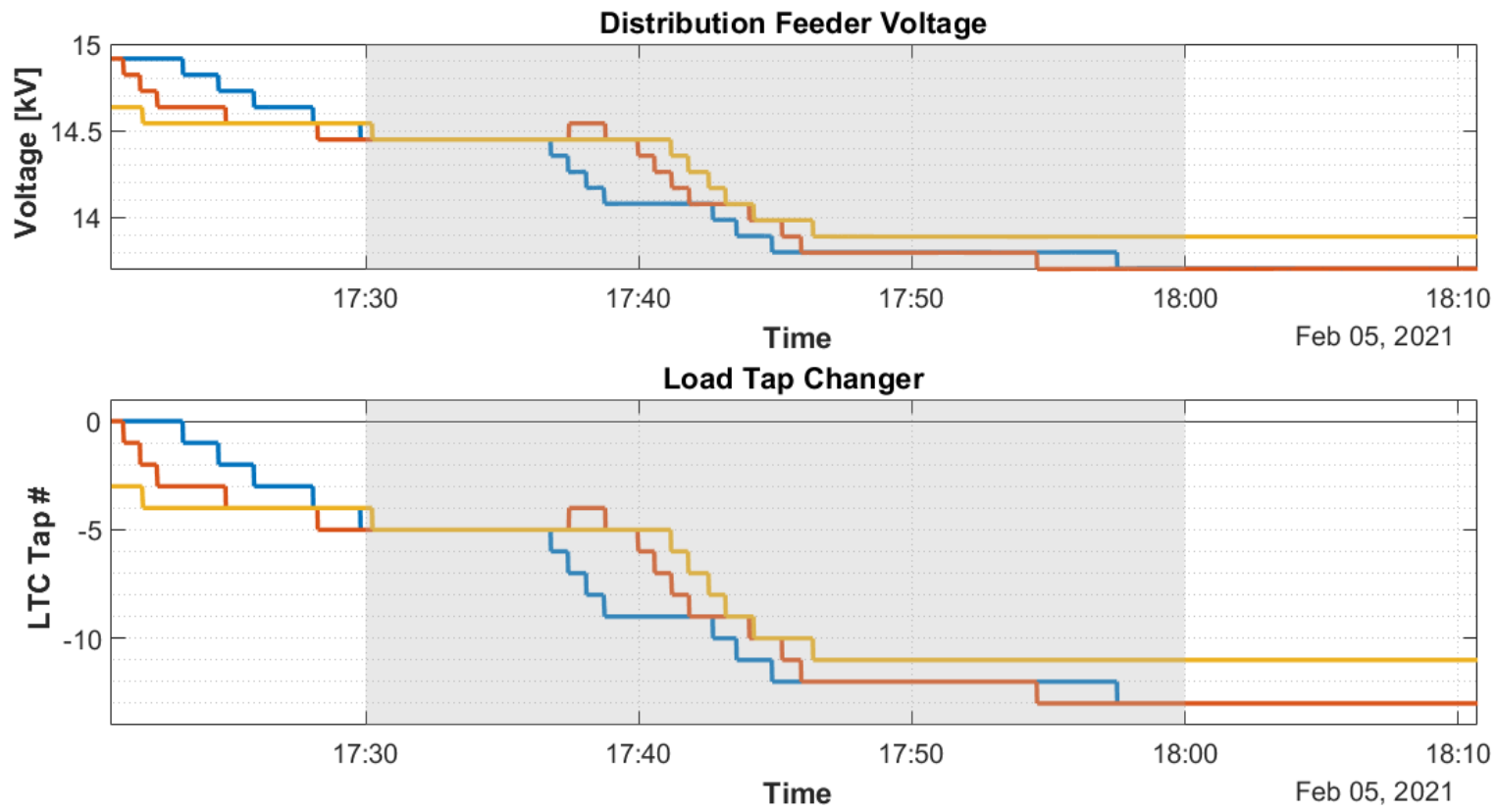

Figure 10. Experimental results for distribution feeder voltages based on change in LTC set point using both DVR and DERMS

Table 4. Summary of Latency Calculations for ADMS Tests

\begin{tabular}{crc}
\hline Scenario & Test Description & $\begin{array}{c}\text { Time to Settle from } \\
\text { Experiment Initialization }\end{array}$ \\
\hline I & Wired Ethernet connection, DVR only & $11 \mathrm{~min}, 23 \mathrm{sec}$ \\
II & Normal wireless, DVR only & $12 \mathrm{~min}, 23 \mathrm{sec}$ \\
III & Weak (attenuated) wireless, DVR only & $13 \mathrm{~min}, 29 \mathrm{sec}$ \\
IV & Wired Ethernet connection, DVR and DERMS & $9 \mathrm{~min}, 33 \mathrm{sec}$ \\
V & Normal wireless, DVR and DERMS & $10 \mathrm{~min}, 37 \mathrm{sec}$ \\
VI & Weak (attenuated) wireless, DVR and DERMS & $13 \mathrm{~min}, 29 \mathrm{sec}$ \\
\hline
\end{tabular}

In Table 4, the attenuated wireless scenarios for both ADMS use cases (scenarios III and VI) resulted in the longest settling times. The shortest settling times were for the baseline wired Ethernet cases (scenarios I and IV). This was consistent for both the DVR-only use case experiments and the DVR-plus-DERMS use case experiments. Note that these are approximate latency calculations based on experiments run at separate times, which could result in tolerance differences between tests. Additionally, there could be minor differences in actual settling time calculations because of noise in the measurements, which can result in fluctuations in LTC tap number measurement (e.g., baseline DVR test, LTC tap measurement fluctuation in Figure 9 between 17:35 and 17:45). 
Using wireless communications can have some impact on DVR and/or DERMS performance within this PLM application. If a data packet were to be missed over the wireless network, this could impact the DVR and/or DERMS processes such that if the signal feedback packets are not received in time, then the ADMS might wait until the next successful data exchange to execute a decision. Based on the results shown in Table 4, the wireless experiments took approximately 12 minutes longer than the wired Ethernet tests. Note that additional tests should be performed with a focus on latency for a more comprehensive comparison among different scenarios in future work. Although the wireless scenarios did take a minute or 2 longer than the wired Ethernet scenarios, there might be a trade-off between cost savings and time to regulate between the wireless and wired Ethernet/fiber applications. Note also that cost was not a focus in this research work, but it would be a useful future study to better understand the trade-offs and benefits of wireless communications for applications such as PLM in distribution systems. 


\section{Conclusions}

The performed experiments demonstrate the capability of evaluating communications latency for a private wireless LTE network under different congestion scenarios. Message prioritization is a unique capability for private LTE networks, which allows some communication traffic to be prioritized over others. Attenuating communication signals is a method to emulate weaker signal strength over a longer distance in a given coverage area within a laboratory setting. This work demonstrates the impact from private LTE communications on latency, and thus the potential impact on a low-latency grid application, such as a DTT scenario of a recloser system.

Measurements show the expected trend of increasing latency for weaker communication links and more traffic congestion. Additionally, results show a considerable difference in realized communication latency when prioritizing traffic used in high-traffic congestion scenarios.

Following the demonstration of the DTT relay experiments, the private wireless LTE network was applied in an ADMS test bed in NREL's Energy Systems Integration Facility. Specifically, the ADMS test bed use case is a study of PLM and voltage regulation within a controllerhardware-in-the-loop evaluation setup. The trends in latency observed from the DTT relaying experiments from Section 2.2 are also demonstrated in Section 3.3; the attenuated wireless scenarios took longer to settle than the baseline wireless and wired Ethernet use cases. These experiments validate the use of private wireless LTE communications in a PLM grid application using two-way communications via a controller-hardware-in-the-loop experimental setup. Note that the system performance is based on feedback to the controller and the operating bandwidth that each controller has, which likely impacted the timing differences observed between the DVR versus DVR and DERMS ADMS scenarios. Possible future work to improve this study includes (1) minor physical modifications (e.g., shortening cables) across the system to ensure the cleanest possible signals to and from each controller, (2) performing a larger number of tests per ADMS and wireless scenario to improve the statistical confidence about the grid performance and latency results, and (3) including an increased number of measurement methods (e.g., Wireshark) to further study the consistency and trends of these results.

The proposed evaluation method for measuring communication timing could also be applied to 5G wireless technologies in future work. Additionally, this study can be repeated with the IEC 61850 communication protocol (rather than Modbus TCP), which would be more realistic in distribution system applications. Some initial studies with the Anterix network using the IEC 61850 communication protocol were performed and are mentioned in the appendix of this report. Future work also includes a more in-depth study of the use of wireless LTE communications in an ADMS, including more realistic real-world aspects, such as cybersecurity impacts on grid performance. 


\section{References}

Aalamifar, F., and L. Lampe. 2016. "Optimized WiMAX Profile Configuration for Smart Grid Communications.” IEEE Transactions on Smart Grid 8 (6): 2723-32.

Achanta, S. V., R. Bradetich, and K. Fodero. 2016. "Speed and Security Considerations for Protection Channels." Presented at the 2016 69th Annual Conference for Protective Relay Engineers (CPRE), 1-9.

Chen, T. M. 2010. "Smart Grids, Smart Cities Need Better Networks [Editor's Note].” IEEE Network 24 (2): 2-3.

Fix, T., et al. 2019. "New Intelligent Direct Transfer Trip Over Cellular Communication." Presented at the 2019 72nd Conference for Protective Relay Engineers (CPRE), 1-9. DOI: 10.1109/CPRE.2019.8765861.

Jawhar, I., N. Mohamed, and J. Al-Jaroodi. 2017. "Networking and Communication for Smart City Systems.” Presented at the 2017 IEEE SmartWorld, Ubiquitous Intelligence Computing, Advanced Trusted Computed, Scalable Computing Communications, Cloud Big Data Computing, Internet of People and Smart City Innovation (SmartWorld/SCALCOM/UIC/ATC/CBDCom/IOP/SCI), 1-7.

Khattak, A. R., S. A. Mahmud, and G. M. Khan. 2012. "The Power to Deliver: Trends in Smart Grid Solutions.” IEEE Power and Energy Magazine 10 (4): 56-64. ISSN: 1558-4216. DOI: 10.1109/MPE.2012.2196336.

Kuzlu, M., and M. Pipattanasomporn. 2013. "Assessment of Communication Technologies and Network Requirements for Different Smart Grid Applications." Presented at the 2013 IEEE PES Innovative Smart Grid Technologies Conference (ISGT), 1-6.

Maitra, A., et al. 2017. "Microgrid Controllers: Expanding Their Role and Evaluating Their Performance." IEEE Power and Energy Magazine 15 (4), 41-49.

Namboodiri, V., et al. 2013. "Toward a Secure Wireless-Based Home Area Network for Metering in Smart Grids.” IEEE Systems Journal 8 (2): 509-520.

Obaidat, M. S., A. Anpalagan, and I. Woungang. 2012. Handbook of Green Information and Communication Systems. Academic Press.

Pacheco, J., C. Tunc, and S. Hariri. 2016. "Design and Evaluation of Resilient Infrastructures Systems for Smart Cities.” Presented at the 2016 IEEE International Smart Cities Conference (ISC2), 1-6.

Schneider, K. P., et al. 2019. "A Distributed Power System Control Architecture for Improved Distribution System Resiliency.” IEEE Access 7, 9,957-70. 
Shariff, F., N. A. Rahim, and W. P. Hew. 2015. "Zigbee-Based Data Acquisition System for Online Monitoring of Grid-Connected Photovoltaic System." Expert Systems with Applications 42 (3): $1,730-42$.

Simms, J., and G. Johnson. 2015. "Protection Considerations for Installation of Distributed Energy Resources." Presented at the 2015 68th Annual Conference for Protective Relay Engineers, 289-301.

U.S. Department of Energy (DOE). 2015. Grid Modernization Multi-Year Program Plan. Washington, D.C. https://www.energy.gov/downloads/grid-modernization-multi-year-programplan-mypp.

Whitehead, D., and R. Smith. 2008. "Cryptography: A Tutorial for Power Engineers.” Presented at the $35^{\text {th }}$ Annual Western Protective Relay Conference, Spokane, Washington.

Institute of Electrical and Electronics Engineers (IEEE). 2018. IEEE Std 1547-2018 - IEEE Standard for Interconnection and Interoperability of Distributed Energy Resources with Associated Electric Power Systems Interfaces. DOI: 10.1109/IEEESTD.2018.8332112.

Zawada, P. J. 2000. "A Survey of Substation Communications Technology." Presented at the 2000 Power Engineering Society Summer Meeting (Cat. No. 00CH37134), 573-78.

Padullaparti, H., A. Pratt, I. Mendoza, S. Tiwari, M. Baggu, C. Bilby, and Y. Ngo. 2021. "Peak Load Management in Distribution Systems Using Legacy Utility Equipment and Distributed Energy Resources.” Presented at the 2021 IEEE Green Technologies Conference (GreenTech).

Survalent. 2020. "ADMS Platform Overview - Survalent: Advanced Distribution Management Systems (ADMS) - SCADA, OMS \& DMS.” Accessed November 11, 2020. https://www.survalent.com/adms-platform-overview/. 


\section{Appendix A. Validation of IEC 61850 Protocol Using Wireless Communications}

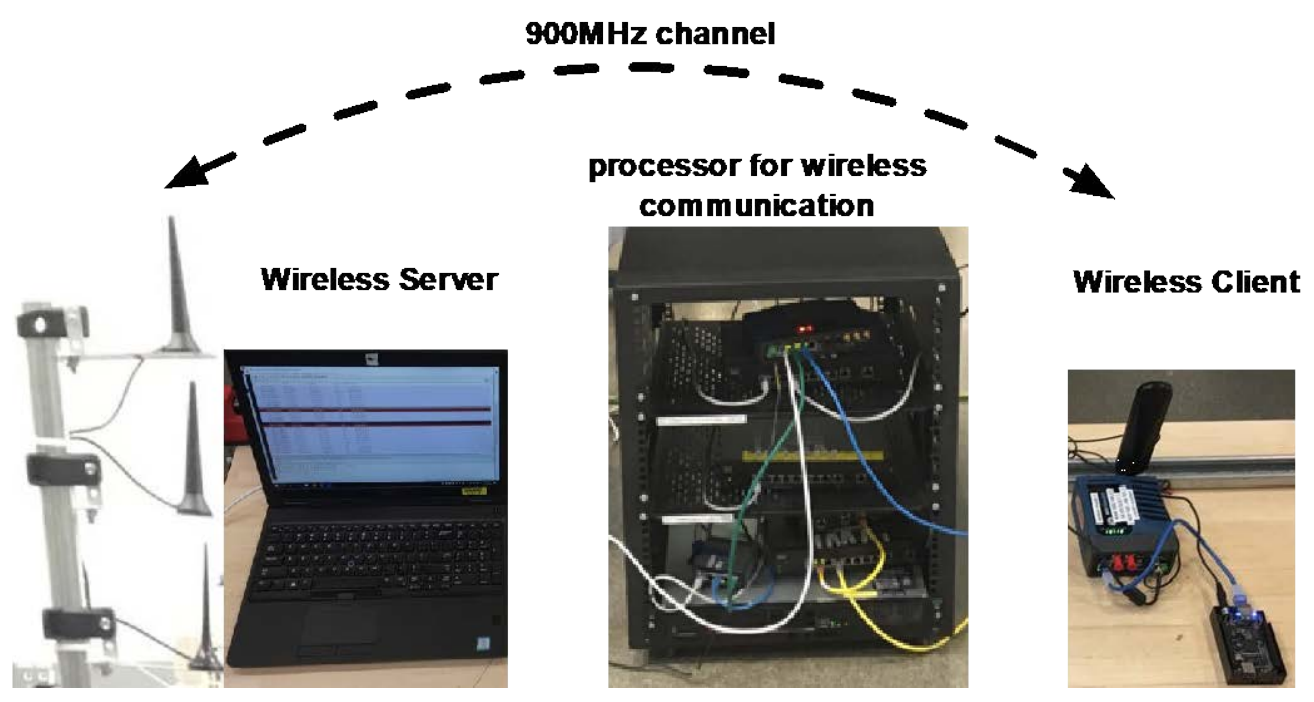

Figure A-1. Experimental setup for server-client communications with IEC 61850 communication protocol over wireless network Photos by NREL

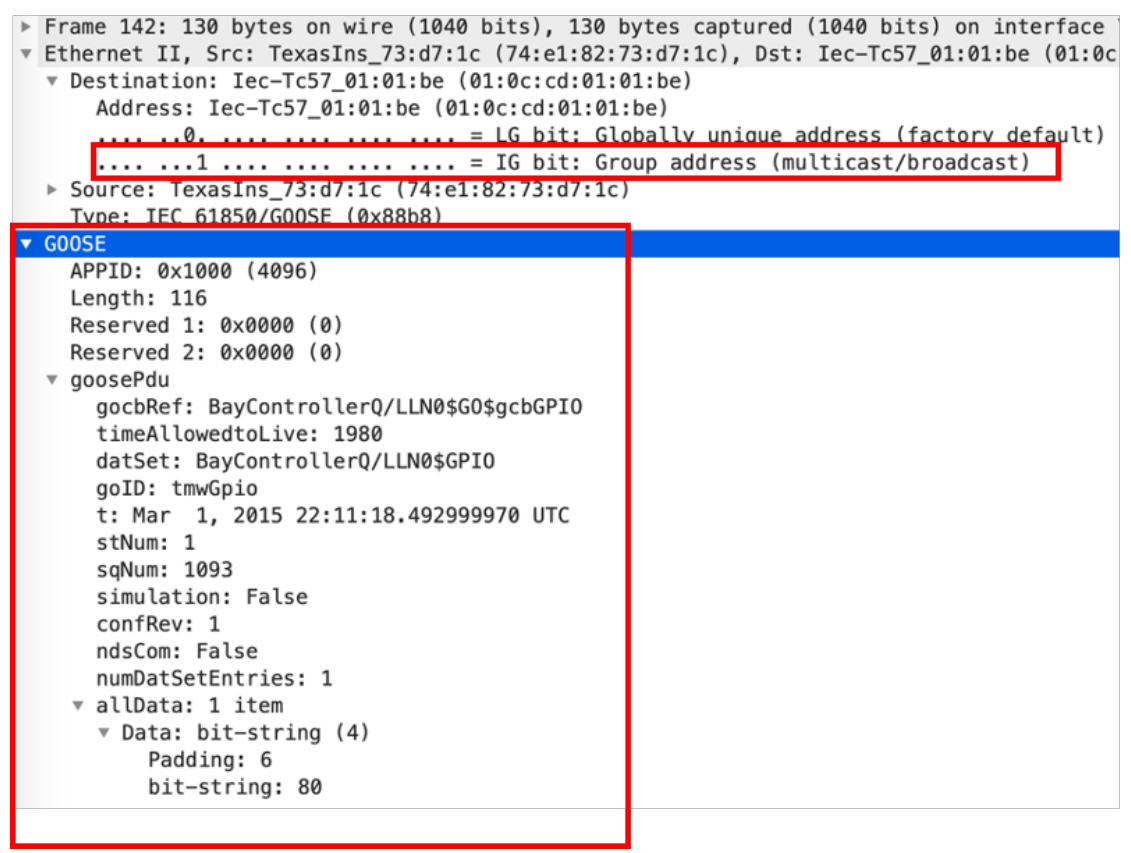

Figure A-2. Wireshark data capture of GOOSE messaging between IEC 61850 Client and IEC 61850 Server IEC over wireless network 


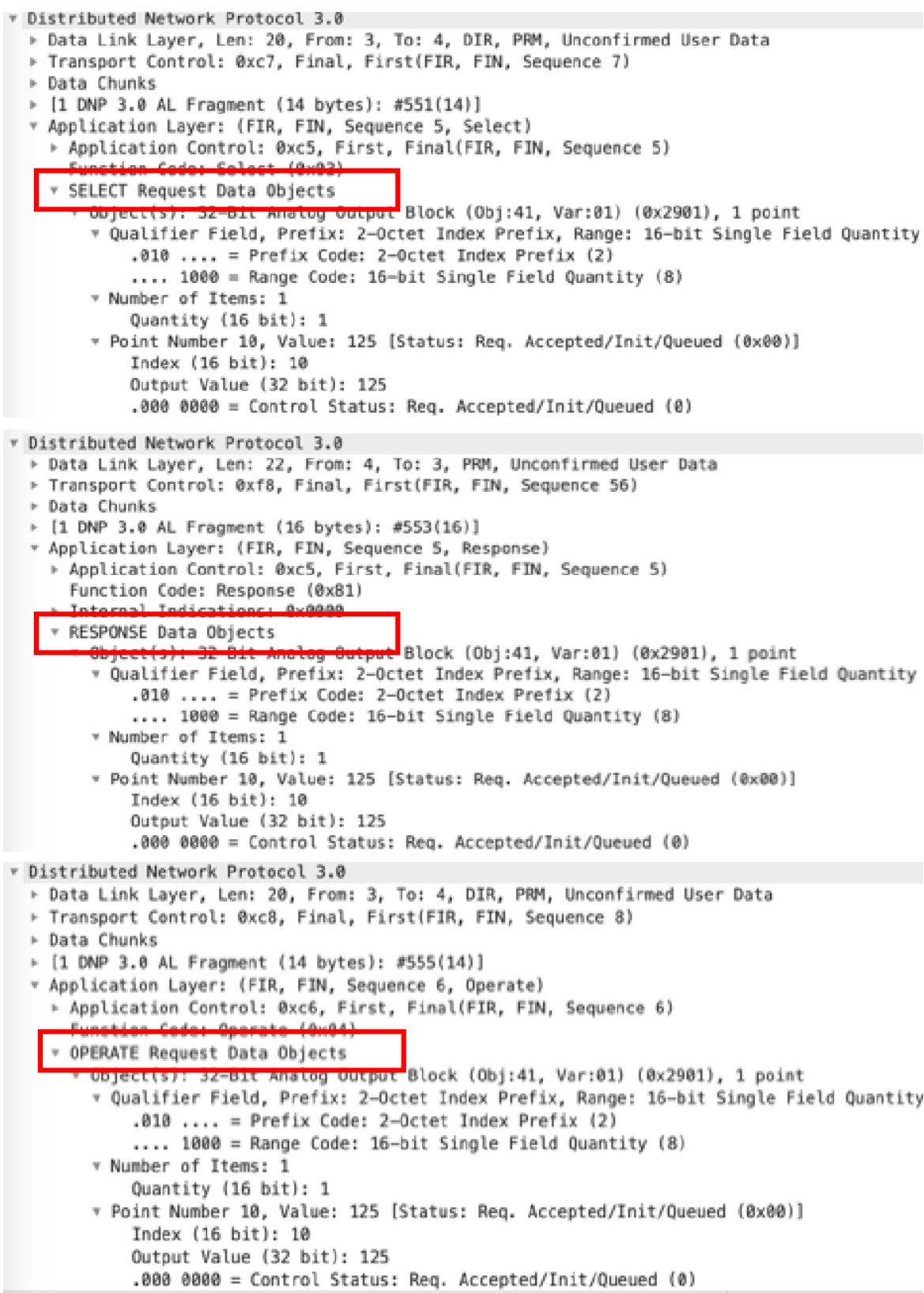

Figure A-3. Wireshark data capture of "select-before-operate" communications using the developed software code to validate IEC 61850 communication protocol over wireless network 